\title{
Quantifying Fidelity for Timed Transition Systems
}

\author{
Sangeeth Saagar Ponnusamy ${ }^{1,2,3}$, Vincent Albert ${ }^{2,3}$ and Patrice Thebault ${ }^{1}$ \\ ${ }^{1}$ Airbus Operations SAS, 316 Route de Bayonne, Toulouse, 31060, France \\ ${ }^{2}$ CNRS, LAAS, 7 Avenue Colonel de Roche, Toulouse, 31400, France \\ ${ }^{3}$ Université de Toulouse, UPS, LAAS, Toulouse, 31400, France \\ \{sangeeth-saagar.ponnusamy,patrice.thebault\}@airbus.com,valbert@laas.fr
}

Keywords: Simulation, Formal Method, Game Theory, Quantitative Reachability, Timed Transition Systems.

\begin{abstract}
The paper addresses one of the fundamental questions in using simulation as a means for system verification and validation, namely, how far the simulation model represents the transition timings of the real system. A formal quantification of this difference in transition timings of a simulation model with respect to the system specification is presented based on game theoretic distance notions from literature. In this two player game, simulation model tries to mimic the system's transitions and incurs a distance if it fails to match the timing of the transition. Fidelity of simulation model is presented through this distance notion based on the quantitative simulation relations and timed simulation game. This game between two timed transition systems is modelled in petri-net formalism and a quantitative reachability graph is generated using TINA tool embedded in ProDEVS simulation platform to explore all such player strategies. The resulting exhaustive exploration yields a global fidelity distribution of the simulation model in terms of transition timings which could be analysed in ProDEVS to gain further insight into the simulation model behaviour with respect to the system model. The approach is demonstrated on a buffer system modelling case study to validate a processor through simulation.
\end{abstract}

\section{INTRODUCTION}

In the development of complex engineering systems, Verification and Validation (V\&V) activities plays a key role in determining the adequacy and fitness for intended use of the systems being designed and developed respectively. These activities necessitate integration of the System Under Test (SUT) with the other systems called environmental systems to perform some test cases and evaluate against criteria such as performance, robustness etc. However, due to realistic limitations such as safety, cost, risk, and availability of systems this is seldom possible and these environmental systems are usually replaced by their models. In certain cases, models of such systems called design models might be available but could not be used due to practical constraints on resources, platform limitations and compositional complexity. Thus it becomes necessary to develop reasonable abstractions of such environmental systems such that the resulting V\&V activity yields same conclusions such as the ones carried out with real systems. This ability of models to replace systems by faithfully reproducing their behaviour is called 'fidelity' and it has been widely discussed in literature (Roza, 1999), (Brade, 2004). There needs to be a metric on this fidelity in order to have acceptable degree of confidence in the V\&V process (Ponnusamy et al., 2014). In this paper, a behavioural fidelity metric for timed systems is discussed based on the quantitative simulation relations proposed in the literature, for example in (Cerny et al., 2010); (Chatterjee and Prabhu, 2015). The broad objective of the paper is, given two timed systems, one being a system specification and other being an abstraction i.e. a (legacy) model, how to quantify the degree of fidelity in terms of transition timings between them for all possible behaviours. In other words, how close (or far) does the model match the event timings of the system for all possible sequence of events.

The paper is structured as follows, a brief overview of simulation fidelity quantification in system V\&V is illustrated followed by quantitative simulation functions for (un)timed systems in section 2. The tool implementation to generate a quantitative reachability is presented in section 3 followed by an application case in section 4 . 


\section{FIDELITY QUANTIFICATION}

An informal description of our approach to fidelity quantification for timed systems is briefly presented before a formal description in section 2.1 and 2.2. Let us consider a $\mathrm{V} \& \mathrm{~V}$ activity where some properties of the SUT, $\varphi_{\text {SUT }}$ are evaluated by stimulating and observing this SUT in conjunction with its environment. In $\mathrm{V} \& \mathrm{~V}$ by simulation, these environmental systems, $M_{\text {sys }}$ are replaced by their models, $\mathrm{M}_{\text {sim }}$ through some abstraction operation, $\alpha$ such as state omission or aggregation. Such abstractions create distance with respect to the real system's behavior called fidelity, $\delta_{\mathrm{F}}$ and it needs to be quantified for all possible behaviors. This is illustrated in figure 1. This quantification is absolute if it is done independent of test cases i.e. some subset of all possible stimulants and relative if it is done with respect to the test cases. In this paper absolute fidelity is discussed and this would intuitively mean that for all possible inputs, the simulation model behaves (within certain bounds) same as that of the system such that the SUT could not see differentiate among them.

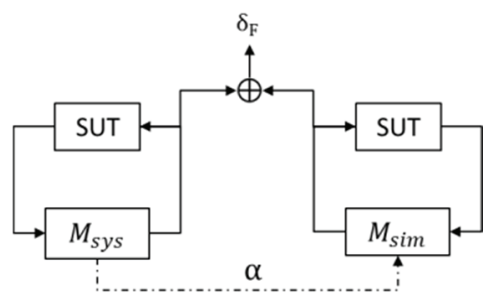

Figure 1: Simulation Fidelity.

Let us consider a system specification given by the system designer and a candidate simulation model as shown in figure below. The dynamics are modeled as a finite labeled timed transition system where for example, from initial state upon receiving a label ' $a$ ' the system moves to the next state in 2 time units and so on.

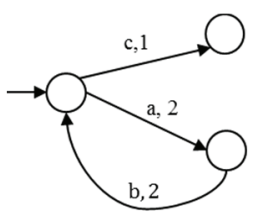

(a) System Model

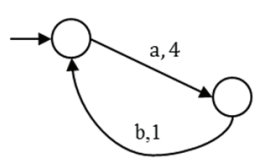

(b) Simulation Model
Figure 2: System \& Simulation Model.

Consider a scenario where the simulation user requires a simulation model with at least $80 \%$ fidelity i.e. it is required to capture the transitions with $80 \%$ (timing) accuracy. For the sake of simplicity, consider the labels of two models are same and they differ only with the time. A model developer, who is tasked with developing or reusing an existing model needs to quantify the model vis à vis this system specification before integrating with other model fragments and deploying on a platform. The objective in this case is to measure the timing difference for each transition and doing for all possible combinations yields a formal fidelity measure. Recalling fidelity is the ability of a model to match every move of the system to the desired degree of accuracy, a two player game can be played between them. In this game the first player also called an attacker plays the role of system whereas the second player also called defender plays the role of simulation model. A model is said to be with sufficient fidelity if the defender wins the game with an acceptable degree of accuracy. In other words, every move of attacker is matched by the defender at the same time. In the given example, first the attacker makes a move with either label ' $c$ ' at $1 \mathrm{~s}$ or ' $\mathrm{a}$ ' at $2 \mathrm{~s}$. For the 'c' move, there exists no counter move by the defender and the game is lost. On the other hand, for the label ' $\mathrm{a}$ ' move by attacker, defender responds with same label in $4 \mathrm{~s}$ and the time cheat is $2 \mathrm{~s}$. For the next move of attacker with ' $b$ ' label at $2 \mathrm{~s}$, defender's response is $1 \mathrm{~s}$ and the time cheat is $-1 \mathrm{~s}$. The net timing error is then $1 \mathrm{~s}$ at the end of two transitions and this error increases linearly for every loop made by the attacker on system model. The resulting timing errors between the corresponding transitions are evaluated against the user requirement at the end to determine the model adequacy. In the next section, these informal game notions are formally presented using the quantitative simulation relations based on two player game theory.

\subsection{Quantitative Simulation Relations}

In (reactive) systems modelling, the behaviour exhibited by the system could be interpreted as a sequence of letters representing observable events collected as a language. A system's behaviour can then be checked against its requirement, both specified as $\omega$ automaton by comparing their languages. This linear view of checking language, also called language inclusion is PSPACE hard for finite state machines (Henzinger, 2013). On the other hand, in a branching time view where the behaviours are captured through tree automata, the algorithmic complexity is only polynomial time. Simulation relations (Alur et al., 1998), which relates two systems based on this branching view, gives a sufficient (but not necessary) condition to check this 
language inclusion between two automata. In this paper, quantitative extensions of classical boolean simulation relations proposed in (Cerny et al., 2010) are used in the context of simulation fidelity i.e. to quantify the degree of similarity between the transition timings of system and simulation model.

Originally intended for software verification where a program implementation is compared against a specification, it is natural to extend this paradigm to the domain of simulation where a model could be interpreted as an implementation of a system specification. This would mean evaluation of all possible behaviours of a system specification against a model i.e. absolute fidelity. In practice, only a subset of the system's state space is explored based on a V\&V plan and only such trajectories need be reproduced by the model with adequate accuracy i.e. relative fidelity. This could be factored in our approach by relatively measuring this distance with respect to the trajectories which are part of the V\&V plan and this is briefly discussed at the end of section 4. It may be noted that a truly absolute measure of fidelity is with respect to the reality which is neither feasible nor useful and hence in our study system specification is assumed correct and approximated to be the real system. In the following section some preliminaries are explained.

\subsubsection{Timed Simulation Relations}

Let us consider the time domain $\mathbb{T}$ with non-negative set of reals $\mathbb{R}_{+}$and over this time domain define the timed automata (Alur and Dill, 1994) is defined by $\mathcal{T}=\left\langle\Sigma, X, T, x^{0}, \delta, R\right\rangle$, where $\Sigma$ is a finite nonempty set of alphabets or labels, $X$ is the finite nonempty set of states, $T$ is a finite set of clocks, $x^{0} \subseteq X$ is the initial non-empty state set, $\delta: X \times \Sigma \times T \rightarrow 2^{X}$ is the transition function and $R \subseteq X$ is the set of accepting states. An accepting run of $\mathcal{T}$ over a finite word $\omega=w_{0} w_{1} \ldots \in \Sigma$ is the sequence of states $x_{0} x_{1} \ldots \in X$ such that $x_{0} \in x^{0}$. Then the language of $\mathcal{T}, \mathcal{L}(\mathcal{T})$ is the set of words accepted by $\mathcal{T}$.

Let us consider two transition systems, $\mathcal{T}_{1}=<$ $\Sigma_{1}, X_{1}, T_{1}, x_{1}^{0}, \delta_{1}>$ and $\mathcal{T}_{2}=<\Sigma_{2}, X_{2}, T_{1}, x_{2}^{0}, \delta_{2}>$, with $\tau_{1} \in \delta_{1}, \tau_{2} \in \delta_{2}$, then $T_{1}$ simulates $T_{2}$ is denoted by $\mathcal{T}_{1} \preccurlyeq_{S} \mathcal{T}_{2}$ and it holds if there exists a binary relation $f \subseteq X_{1} \times X_{2}$ such that if $\left(x_{1}, x_{2}\right) \in f$ then

$$
\forall\left(x_{1}, \tau_{1}, x_{1}^{\prime}\right) \exists\left(x_{2}, \tau_{2}, x_{2}^{\prime}\right) \text { such that }\left(x_{1}^{\prime}, x_{2}^{\prime}\right) \in f
$$

and it becomes bisimulation, $T_{1} \approx_{B S} T_{2}$ when

$$
\forall\left(x_{2}, \tau_{2}, x_{2}^{\prime}\right) \exists\left(x_{1}, \tau_{1}, x_{1}^{\prime}\right) \text { such that }\left(x_{1}^{\prime}, x_{2}^{\prime}\right) \in f
$$

These simulation relations are usually boolean i.e. a simulation model either simulates the system or not.
Quantitative extensions of these boolean notions are based on finite-state turn based two player game graphs. Two player game theoretic notions have been used in verification as well as synthesis perspectives in the formal modeling and analysis of systems (Henzinger, 2013).

\subsubsection{Timed Simulation Games}

The two player turn based game is briefly introduced in this section followed by the game between the system and simulation model in the context of quantifying its degree of similarity i.e. fidelity. A game graph is a tuple, $g=<X, X^{1}, X^{2}, E, x_{0}>$ where $X$ a finite set of states is partitioned as $X^{1}$ and $X^{2}$ for the first and second player respectively such that $X^{1} \cup X^{2}=\mathrm{X}, X^{1} \cap X^{2}=\varnothing, E \subseteq X \times X$ is the set of edges, $x_{0}$ is the initial state of the play (Alur et al., 1998). The dynamics of the transition system described by its states and transitions are interpreted as nodes i.e. states and edges of this game.

The untimed game starts from state $x_{0} \in X$ with a player 1 making the move to $x_{1} \in X^{1}$ to which the player 2 counters by making a move $x_{2} \in X^{2}$. The first play is over now and the game is started again. At the end of first play, if the player 2 cannot match player 1's move it is allowed to cheat (Cerny et al., 2010) and in doing so incurs a penalty and there are different ways of measuring this cheat such as weighted mean etc. This cheat measure gives a metric on the degree of similarity between two models and used in generating quantitative reachability for untimed labelled transition systems in (Ponnusamy et al., 2016). In this case of untimed or time-abstract fidelity games, player 1 plays on the system model and player 2 plays on the simulation model. Every move on the system model by the first player is followed by the second player on simulation model and this continues until one wins. In particular, a simulation relation exists if player 2 always has the winning strategy. The strategy of the player to choose each move may or may not depend on the history of previous moves and in this paper we employ the memory-less strategy. The set of visited states in the game is called a play which is denoted by $\rho=\rho_{1} \rho_{2} \ldots$ and this is akin to the path of a transition system or trace if there is a propositional evaluation at each such state.

However, in timed game, the turn based nature of the game does not strictly hold true due to the temporal nature. The evolution of player 1 is independent of the player 2 since the objective of player 2 is to match player 1 timings. In other words, player 2 is not allowed to win by infinitely blocking 
the player 1's turn (Chatain et al., 2009) whereas it wait until player 1 finishes its turn.

\section{Proposition 1: Player 1 can block player 2's time}

Let us assume a system and simulation model in the figure 3 and recall player 1 plays on $M_{\text {sys }}$ whereas player 2 plays on $M_{\text {sim }}^{1}$.

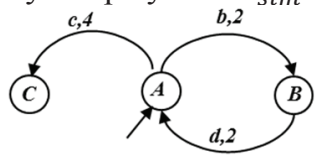

(a) $M_{\text {sys }}$

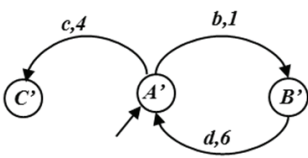

(b) $M_{\text {sim }}^{1}$
Figure 3: Blocking Game.

In this case, without blocking, player 2 label ' $b$ ' is fired earlier and if player 1 moves ' $c$ ' instead, there is a cheat whereas in reality the player 2 does not cheat for ' $c$ ' transition. The blockage of time helps to avoid this problem. Intuitively, a simulation model has to mimic system model so it has to see what the system does first or else it may end up in cheating even if a way not to cheat exists.

\section{Proposition 2: Player 2 cannot block player 1's time}

This assumption, also found in literature (Chatain et al., 2009), could be explained with the following example of game between a system and simulation model in the figure below, (a) $M_{\text {sys }}$

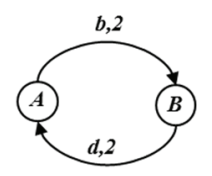

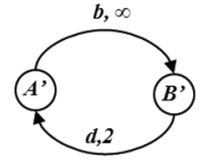

(b) $M_{\text {sim }}^{1}$

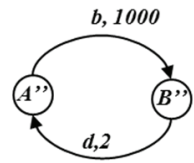

(c) $M_{\text {sim }}^{2}$
Figure 4: Non-blocking Game.

In this case, the third model is a better approximation of the first model than the second. However, if the game is played for $<1002$ time units both the simulation models are deemed unfit and the system model cannot move further from state B. This can be mitigated by segregating the evolution of system model from that of simulation model. In such case, the time difference is 998 time units for the third model and $\infty$ for the second model.

Then, formally, the game between system, $\mathrm{M}_{\text {sys }}$ and simulation model, $\mathrm{M}_{\text {sim }}$ is denoted by $g\left(M_{\text {sys }}, M_{\text {sim }}\right)$ with the state space, $X_{\text {sys }} \times X_{\text {sim }}$. Let $\sigma_{i}^{1,2}$ be label and $t_{i}^{1,2}$ be associated transition time and of player 1 and 2 respectively at play $i, \tau_{\text {sys }} \in$ $\delta_{\text {sys }}$ and $\tau_{\text {sim }} \in \delta_{\text {sim }}$, player actions of selecting a transition from one model and handing over the turn to other player i.e. enabling transition of the other model are denoted by $p_{1}: \tau_{s y s} \rightarrow \tau_{\text {sim }}$ and $p_{2}: \tau_{\text {sim }} \rightarrow \tau_{\text {sys }}$. For a given play of positive integers, $i \in \mathbb{I}_{+}$, player 1 move is defined as follows,

$$
\left(\mathrm{x}_{\text {sys }}, \tau_{\text {sys }}, \mathrm{x}_{\text {sim }}\right) \stackrel{\mathrm{p}_{1}}{\rightarrow}\left(\mathrm{x}_{\text {sys }}^{\prime}, \tau_{\text {sim }}, \mathrm{x}_{\text {sim }}\right)
$$

with the transition time of simulation model

$$
\mathrm{t}_{\mathrm{i}}^{2}=\mathrm{t}_{\mathrm{i}}^{2}+\mathrm{t}_{\mathrm{B}_{i}} \mid \mathrm{t}_{\mathrm{B}_{i}}=\mathrm{t}_{\mathrm{i}}^{1} \quad \text { if } \mathrm{t}_{\mathrm{i}}^{1}>\mathrm{t}_{\mathrm{i}}^{2}
$$

where $t_{B}$ is the blocked time for player 2 . Then the player 2 move is defined as

$$
\left(x_{\text {sys }}^{\prime}, \tau_{\text {sim }}, x_{\text {sim }}\right) \stackrel{p_{2}}{\rightarrow}\left(x_{\text {sys }}^{\prime}, \tau_{\text {sys }}, x_{\text {sim }}^{\prime}\right) \text { if }\left\{\begin{array}{c}
\sigma_{i}^{1}=\sigma_{i}^{2} \\
t_{i}^{2} \leq t_{i+1}^{1}
\end{array}\right.
$$

The play is terminated if $\sigma_{i}^{1} \neq \sigma_{i}^{2}$ regardless of their transition times and the player 1 is deemed won. In all other cases, the next play, $i+1$, is started with player 1 move if $t_{i}^{2}>t_{i+1}^{1}$. At the end of each completed play, the time difference between the corresponding transitions i.e. labels, $\Delta \mathrm{t}_{\mathrm{i}}$ is calculated using,

$$
\Delta \mathrm{t}_{\mathrm{i}}=\left(\mathrm{t}_{\mathrm{i}}^{2}-\sum_{\mathrm{n}=1}^{\mathrm{i}} \mathrm{t}_{\mathrm{B}_{i}}\right)-\mathrm{t}_{\mathrm{i}}^{1}
$$

It may also be seen that such error function being a directed metric (Chatterjee and Prabhu, 2015) satisfies the reflexivity and triangular inequality i.e. for all, $\Delta \mathrm{t}\left(\mathcal{T}_{1}, \mathcal{T}_{1}\right)=0$ and $\Delta \mathrm{t}\left(\mathcal{T}_{1}, \mathcal{T}_{3}\right) \leq \Delta \mathrm{t}\left(\mathcal{T}_{1}, \mathcal{T}_{2}\right)+$ $\Delta \mathrm{t}\left(\mathcal{T}_{2}, \mathcal{T}_{3}\right)$ respectively. This helps in incremental model development and assembly with bounded timing error on the resulting composition.

The timing error quantification through this game based approach can be extended to system and/or simulation models whose transition timings are not defined precisely but in an interval as well. Let us define such interval for the system and simulation model as $\left[t^{1,2^{l b}} t^{1,2^{u b}}\right]$ where $l b$ and $u b$ refers to lower and upper bounds on transition timings. In this case, intuitively the interval difference is the timing difference and Eq.4 becomes,

$$
\mathrm{t}_{\mathrm{i}}^{2^{l b}}=\mathrm{t}_{\mathrm{i}}^{2^{l b}}+\mathrm{t}_{\mathrm{B}_{i}} \mid \mathrm{t}_{\mathrm{B}_{i}}=\mathrm{t}_{\mathrm{i}}^{\mathrm{l}^{l b}} \quad \text { if } \mathrm{t}_{\mathrm{i}}^{1^{l b}}>\mathrm{t}_{\mathrm{i}}^{2^{l b}}
$$

where $t_{B}$ is the blocked time for player 2. In other words, the transition of player 2 is enabled once player 1's lower bound transition time is enabled. Then the interval timing error, $\left[\Delta t_{i}^{l b} \Delta t_{i}^{u b}\right]$ is calculated as,

$$
\begin{aligned}
\Delta t_{i}^{l b} & =\left(\mathrm{t}_{\mathrm{i}}^{2^{l b}}-\sum_{\mathrm{n}=1}^{\mathrm{i}} \mathrm{t}_{\mathrm{B}_{i}}\right)-\mathrm{t}_{\mathrm{i}}^{1^{l b}} \\
\Delta t_{i}^{u b} & =\left(\mathrm{t}_{\mathrm{i}}^{2^{u b}}-\sum_{\mathrm{n}=1}^{\mathrm{i}} \mathrm{t}_{\mathrm{B}_{i}}\right)-\mathrm{t}_{\mathrm{i}}^{1^{u b}}
\end{aligned}
$$


However, such interval error quantification needs to be further studied and is not yet implemented in our tool and only transitions fired at punctual time i.e. $t^{1,2^{l b}}=t^{1,2^{u b}}$ is considered in this study.

In discussing fidelity quantification through such game based approach, one of the key difficulties is exploring the player's strategies. Instead of a simulation approach, which is semi-formal and often error prone, a formal method of exploring all such player strategies is needed. In this context, a reachability graph generation which explores all the player's strategies to quantitatively determine the corresponding transition timings is presented in the next section.

\subsection{Quantitative Reachability}

Reachability, in general, is the problem of determining the existence of a trajectory that visits a state. An exhaustive exploration of all such trajectories results in a reachability set, $\mathfrak{K}$ which is usually verified against some boolean specification such as safety and this process is called model checking. Since timed games generate a quantitative measure for each trace of the simulation model vis à vis the system model, generating a reachability set of these timed games result in a formal and quantifiable reachability set. In other words, this is an exhaustive exploration of all the player strategies. However, in contrast to untimed games, continuous evolution of time for the attacker and blocking for the defender need to be taken into account in the play and error quantification as well.

A key benefit of such formal approach is the global distribution of fidelity in terms of event timings with respect to the system model. Such quantitative graphs can be analyzed to determine the optimal strategies, least or maximum error paths etc. In practice, simulation models are usually not developed from scratch but built by reusing the existing model fragments from a library. In such cases, the global distribution of fidelity could be analyzed to determine the adequacy of a particular model for the given test case.

The (pseudo) reachability graph is illustrated in figure 5 for games described in section 2 and figure 2. The transitions of attacker and defender are given in solid and dotted arrows respectively. The first play is over at $6 \mathrm{~s}$ and the second play is over at $7 \mathrm{~s}$ and it can be seen that the blocking time is 2 s. In addition, due to the absence of a matching transition for attacker move on 'c' label, the game is locally lost on this path.

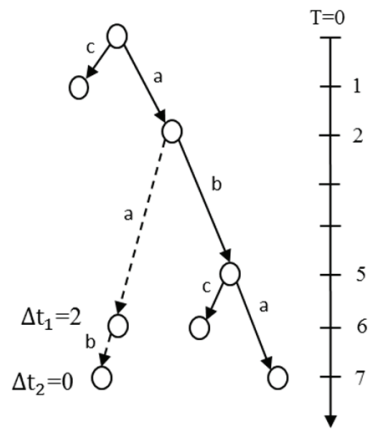

Figure 5: Quantitative Timed Reachability Graph.

Then the timing error is calculated as $\Delta t_{1}=(6-2)-$ $2=2$ and $\Delta t_{2}=(7-2)-5=0$ and so on. It can be easily seen that the pair-wise timing error can be deducted from this aggregate time, for example, the timing difference for second turn is -2 . Such evolution can be analyzed and visualized for better understanding of the model fidelity.

\section{IMPLEMENTATION}

The game semantics and reachability generation discussed in section 2 is implemented in the Petri-Net formalism. Timed petri-net is an extension of classical petri-net formalism (Berthomieu and Diaz, 1991) with firing time for the events. Such formalism is widely used to represent the timed execution of discrete event systems interleaved with (possibly zero) delays. Formally, a petri-net is a tuple

$$
M=<P, \tau, A, w, I_{s}, p_{0}>
$$

where

- $\mathrm{P}$ is a finite set of symbols called places

- $\tau$ is a finite set of symbols called (timed) transitions with $\mathrm{P} \cap \tau=\emptyset$

- $A \subseteq(\tau \times P) \cup(P \times \tau)$ is the set of arcs defining the flow relation

- $w: A \rightarrow \mathrm{N}$ is the function defining the respective weights of the arcs, $\mathrm{N}=1$ in our case

$-I_{s}: \tau \rightarrow I^{+}$is static interval function with $I^{+}$, the non-empty set of positive real intervals including 0 .

$-p_{0}: P \rightarrow \mathrm{N}$ is the initial marking

Informally a transition, $\tau$ is enabled if there is a token at the corresponding place, $p \in P$ and moves to the next state defined by the flow relation. This token and place formalism of Petri-net is amenable to model the two player turn-based game which is alternating in terms of player turns. In the current study no concurrency is assumed and the resulting games have only total states. A state $s$ of a Petri net is a couple < $m, I>$ where $m$ is the marking and I is the interval 
function, $I: \tau \rightarrow I^{+}$which associates to each enabled transition at marking $m$ a temporal interval. In addition, only intervals under the form $[\theta, \theta]$, i.e. deterministic event timings are considered although firing at timings drawn randomly from uniform distribution is also possible.

\subsection{Tool Implementation}

The game semantics described in previous sections has been implemented in TINA, a (un)timed petri-net editor and analysis tool used to generate marked reachability graph, $\mathfrak{K}^{\mathfrak{g}}$ for timed systems through state classes (Berthomieu and Diaz, 1991). This game semantics is then integrated into ProDEVS, a simulation platform for systems modeled in Discrete EVent Specification (DEVS) formalism, (un)timed classical automata and untimed interface automata $(\mathrm{Vu}, 2015)$. The system and simulation models are constructed as timed automata in ProDEVS. In addition, it may be noted that DEVS is akin to timed interface automata which is essentially finite state automaton with embedded time and differentiation between input and output labels. DEVS, whose definitions can be found in (Zeigler et al., 2000) is a hierarchical and modular formalism used to model discrete, continuous and hybrid timed transition systems. Since we intend to extend the current quantitative approach to timed interface automata from the existing timed automata, models are constructed in ProDEVS itself. These models are then converted to equivalent timed petri-net models in TINA. The game is automatically constructed between them in TINA$\mathrm{ND}$ editor and the reachability graph is generated. It may be noted that since petri-net simulator per se does not handle data, these are encoded as guards and actions on the transitions through associated $\mathrm{c}$ files to generate dll files to be run by TINA. This graph in text form is then parsed in ProDEVS to perform some analytics for better understanding and visualization. The ProDEVS parser constructs a reachability tree which can then be visualized and plots the evolution of cheats along the play, distribution of cheats etc. The replay feature allows to choose a particular cheat from the cheat distribution plot to see the associated path to better understand when and where the simulation model behaviour differs with respect to the system.

The overall methodology is illustrated in the figure below.

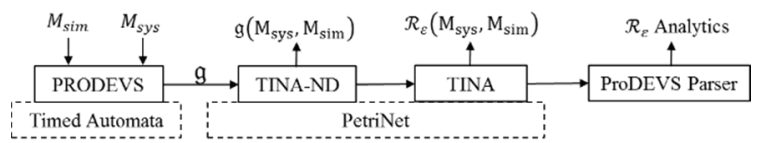

Figure 6: Implementation.
It can be seen that the modeling and parsing are done in ProDEVS with rest being in TINA. Alternatively, modeling and reachability generation could be done directly in TINA as well and ProDEVS could then be used to simply parse the data. It may be seen that, given a system design model and a simulation model, the game is constructed automatically and the resulting output is exhaustive timing error quantification over all possible transitions. The simulation user or the developer may then decide to improve the simulation model or relax the $\mathrm{V} \& \mathrm{~V}$ requirements. This approach, apart from quantifying the global fidelity independent of V\&V objectives, is also useful in iteratively refining the design with respect to $\mathrm{V} \& \mathrm{~V}$ scenarios especially in the early system development when the design is not frozen.

\section{APPLICATION CASE}

The application case considered is a simple FIFO buffer which is connected to a job generator and a processor. The buffer system model is shown in figure 7 where it receives the job, $e 0$ from generator and sends it to the processor, $s 0$ with the associated number of jobs stored in a queue variable, $q$. In addition, the buffer sends the job based on the processor status, el. Let the processor be the SUT under some user defined scenarios, $\varphi_{p=1 \ldots P}$. This scenario of experimentation is illustrated using the experimental frame formalism (Zeigler et al., 2000) where the SUT and the environment systems such as buffer and generator need to perform this validation activity could be seen. In addition, such experimental frame may contain transducer and acceptor which are used for interpretation and validation of experimental frame component's outputs. For example, a transducer might convert processor status as number of processed jobs which will then be compared against the generated jobs.

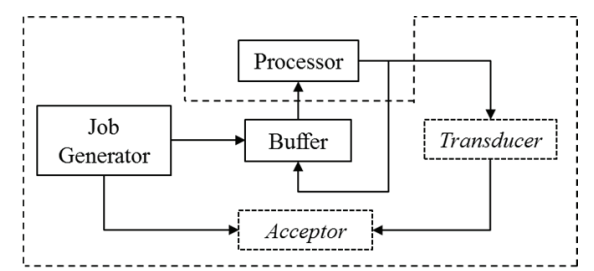

Figure 7: Buffer Model for Processor Validation.

In validating the buffer through simulation, a model of its environment, in this case the buffer, need to be modelled with a quantifiable degree of fidelity. Let us consider the buffer system specification, $M_{\text {sys }}$ 
and two simulation models of the system, $M_{\text {sim }}^{1,2}$ as shown in figure 8 and 9 respectively.

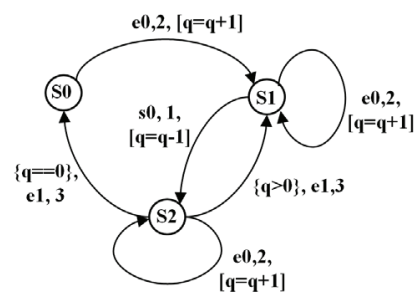

Figure 8: Buffer System Model.

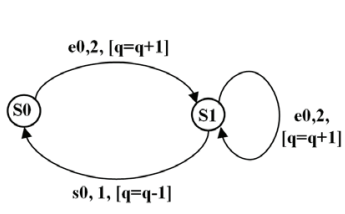

(a) $M_{\text {sim }}^{1}$

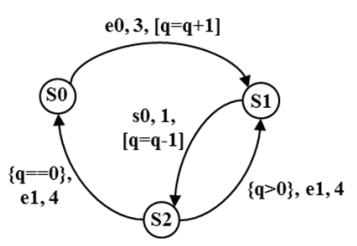

(b) $M_{\text {sim }}^{2}$
Figure 9: Buffer Simulation Model.

The transition labels are typically given in the form of tuple $<\{\}, \sigma, \mathrm{t},[]>$ where \{\}$,[]$ refers to guards and actions respectively. In this case, the guards and actions are on the queue variable, $q$.

This game can be either played state bounded or equivalently play bounded. In the former, the maximum number of state classes generated during reachability construction is fixed whereas in the latter the play is terminated only if all the winning trajectories (if it exists) where $\sigma_{i}^{1}=\sigma_{i}^{2}$ of player 2 are played. In addition, a play can be terminated prematurely if the number of lost trajectories exceeds a certain user defined bound. Different such techniques could be employed to manage the game and interpret the results to determine the fidelity according to the user requirement. In the following section some fidelity metrics are discussed for the buffer model.

\subsection{Analysis Results}

The timed fidelity game is played between $M_{s y s}$ and $M_{\text {sim }}^{2}$ and a quantitative reachability graph is generated for a maximum $10^{3}$ state classes. Since the size of $\mathfrak{K}^{\mathfrak{g}}$ is limited, the first question is how many traces are generated and how long they are i.e. length. In total 4661 traces were generated with 3640 traces has maximum trace length of 26 transitions. It may be reminded that in this case, the system model makes infinite number of turns regardless of the simulation model and incompleteness of each trace is predominantly due to the truncation of reachability states generated. The distribution of all such transitions can be visualised in figure 10 . It can be seen that most traces have one or two transitions empty due to reachability graph truncation and this information can be used to limit or extend the limit of exploration.

For each trace, the number of plays may be different i.e. a play might be lost but still the trace contains only player 1 's transitions. It can be seen from figure 11 that simulation model can match the transition labels for a maximum of 5 plays for 45 traces. For each of these traces, associated timing error can be extracted similar to figure 5 .

For example, the trace with transition sequence $e 0 \stackrel{2}{\rightarrow} s 0 \stackrel{1}{\rightarrow} e 1 \stackrel{3}{\rightarrow} e 0 \stackrel{2}{\rightarrow} s 0 \stackrel{1}{\rightarrow} e 1$ of system model can be matched by the corresponding sequence $e 0 \stackrel{3}{\rightarrow} s 0 \stackrel{1}{\rightarrow} e 1 \stackrel{4}{\rightarrow}$ $e 0 \stackrel{3}{\rightarrow} s 0 \stackrel{1}{\rightarrow} e 1$ of the simulation model and the net timing error is 3 time units at the end of fifth play. However, for some other traces it can match only partially, for example one can intuitively see that a job can arrive at any state for the system model whereas the simulation model can take job only at state $\mathrm{S} 0$. In such traces, (e.g. $e 0 \stackrel{2}{\rightarrow} e 0 \stackrel{2}{\rightarrow} s 0 \stackrel{1}{\rightarrow}$... by the system) the game is partially lost and such information too can be obtained.

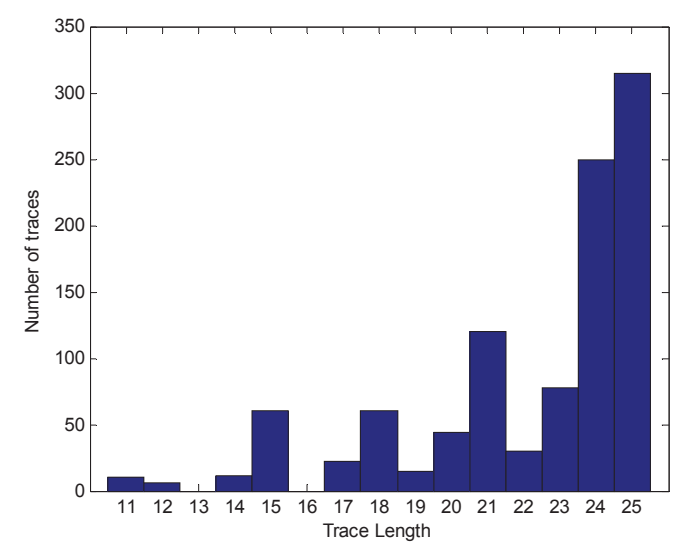

Figure 10: Trace length vs Number of traces.

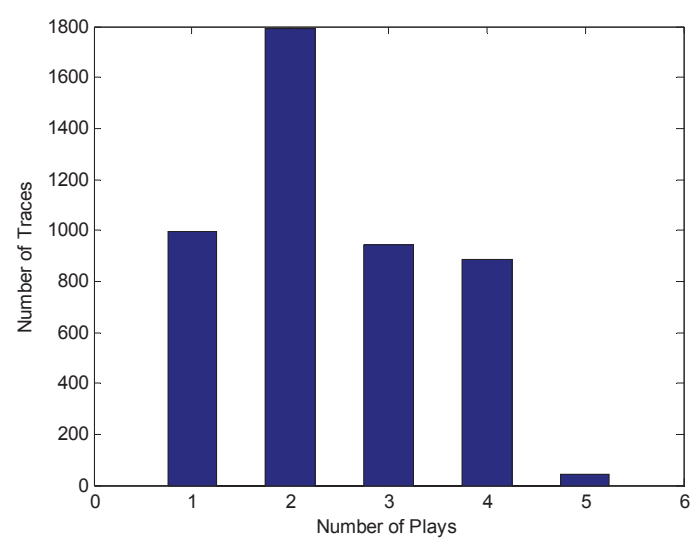

Figure 11: Total number of plays distribution. 


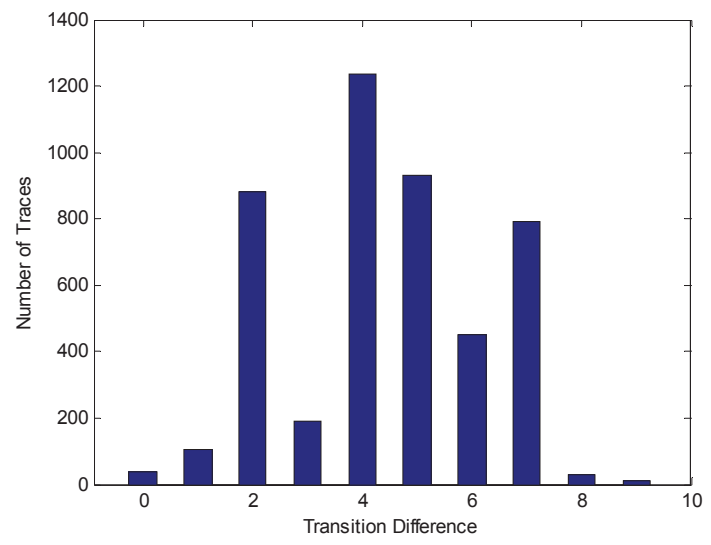

Figure 12: Transition difference distribution.

Another key information of interest is the lead information i.e. how far the system is in advance before the simulation model and this represents the overall lag of the simulation model with respect to system. A near perfect simulation model has less lag and increase in lag is either due to the play being lost in that trace especially for systems with loops such as buffer or simulation model timings are higher. The figure 12 shows this difference and it can be seen that almost all lag is due to the play being lost in corresponding traces. At maximum only one transition i.e. $\mathrm{e} 0$ is matched for 995 traces.

A key aspect which is not discussed is the role of $\mathrm{V} \& \mathrm{~V}$ objectives in this fidelity quantification. In the current study, all the differences in transition timings are equally weighed. However, in reality a model is developed with some V\&V objectives behind and in such cases some transitions are of more interest than the others. Let us consider a requirement, $\varphi_{1}$ on SUT stating that all the sent jobs must be processed by the processor i.e. no job is lost. In other words, an ideal buffer must store and send the jobs to processor as a function of processor status. In case of first simulation model this is not true as the processor status is not modeled. This is characterised by the losing game in the third play of the game whenever the system makes a move with el label. However, in case of second simulation model the game is not lost but the event timings are different. On the other hand, consider requirement, $\varphi_{2}$ on SUT stating processor expects at least one job at delivered by the buffer at $3 \mathrm{~s}$ and in this case first simulation model matches exactly the transition timings $e 0 \stackrel{2}{\rightarrow} s 0 \stackrel{1}{\rightarrow} e 0$ compared to the $M_{\text {sim }}^{1}$. Thus, depending on the requirement, some transition timings are weighed more with weighting $w_{1}$, than the others with weighting $w_{2}$, in which case the complexity of the method is increased to $\mathcal{O}\left(\left|w_{1}\right|\left|w_{2}\right||X|^{3}|E|\right)$.

\section{OUTLOOK \& CONCLUSIONS}

A formal quantitative approach to simulation fidelity based on simulation relations and two player game is presented. Our contribution is threefold, first, extending timed games into a fidelity problem, mapping this game in petri-net formalism, generation of quantitative reachability and analysis with some fidelity metrics. However, this explicit enumeration of traces along with their (timing) distances may suffer from the curse of dimensionality and of limited use in large scale systems. This may be mitigated by using efficient data structures such as using Binary Decision Diagrams (BDD) and studies need to be made in abstraction, and abstraction refinement techniques, especially for continuous systems. Another practical challenge is the availability of the system specification, especially in formal language such as timed automata. Even in case of such availability, there could be interoperability issues between the modelling formalisms used by the model developer and the system designer. In addition, the current study concerns only timed automata which does not differentiate between the labels i.e. inputs and outputs and does not capture the environment assumptions. This study is currently being extended to interface automata and an untimed distance notion for interface automata (Cerny et al., 2014) has been implemented in ProDEVS. An extension to timed interface automata is being studied which will enable fidelity quantification of the DEVS systems.

The quantitative perspective discussed in the paper will enable different stakeholders in the system $\mathrm{V} \& \mathrm{~V}$ process to develop and reuse models with a known and assured level of fidelity. For example, the model developer could gain key insights into the model behaviour and chooses the best abstraction of the system vis à vis the scenario. On the other hand, the system test team would have a measure of fitness on the models being used for the V\&V which would mitigate unfeasible or unclear model fidelity requirements. In addition, this would benefit the system designer in making improvements or modifications to the system model. These benefits would allow not only to select a consistent model with sufficient level of fidelity according to the test case with different criteria such as performance, robustness etc. but also to help in quantifying the fidelity of the overall V\&V process. Such a quantitative framework to fidelity will enable significant benefits in avoiding redundant modelling and validation effort thereby saving cost and time in product development especially in replacing real tests with simulation i.e. virtual testing. 


\section{ACKNOWLEDGEMENTS}

The authors would like to thank Bernard Berthomieu of CNRS, LAAS for his contribution in using FIACRE and TINA for this study.

\section{REFERENCES}

Albert, V., Ponnusamy, S.S., 2016, Encoding CDEVS and PDEVS into Timed Petri Net: theory and application, Journées DEVS Francophones, Accepted.

Alfaro, L., Faella, M., Stoelinga, M., 2009, Linear and Branching System Metrics, IEEE Trans. Software Eng, Vol 35(2), 258-273.

Alur, R., Henzinger, T., Kupferman, O., Vardi, M., 1998 Alternating refinement relations. Lecture Notes in Computer Science, Vol 1466, 163-178.

Alur, R., and Dill, D., 1994, A theory of timed automata, Theoretical Computer Science, Vol 126,183-235.

Brade D, VV\&A Global Taxonomy (TAXO), 2004, Common Validation, Verification and Accreditation Framework for Simulation, REVVA.

Berthomieu, B., Diaz, M., 1991, Modeling and verification of time dependent systems using time Petri nets, IEEE Trans on Software Engineering, Vol. 17, no. 3, 259273.

Cerny,P., Henzinger, T. \& Radhakrishna, A., 2014, Interface simulation distances. Theoretical Computer Science, Vol 560, 348-363.

Cerny,P., Henzinger, T. \& Radhakrishna, A., 2010, Simulation Distances, Lecture Notes in Computer Science, Vol 6269, 253-268.

Chatain, T., David, A., Larsen, K.G., 2009, Playing games with timed games, Proceedings of the 3rd IFAC Conference on Analysis and Design of Hybrid Systems, Zaragoza, Spain.

Chatterjee, K., Prabhu,V.S., 2015, Quantitative Temporal Simulation and Refinement Distances for Timed Systems, IEEE Transactions on Automatic Control, Vol 60, Issue 9, 2291-2306.

Girard, A., Pappas, G.J., 2007, Approximation Metrics for Discrete and Continuous Systems. IEEE Transactions on Automatic Control, Vol 52, Issue 5, 782-798.

Henzinger, T. A., 2013, Quantitative reactive modeling and verification, Journal of Computer Science Research and Development, Vol 28 Issue 4, 331-344.

Ponnusamy, S.S., Thebault P., Albert V., 2016, Simulation Fidleity-A Game Theoretic Approach, Spring Simulation Multi-Conference, US, Accepted.

Ponnusamy S.S., Albert V., Thebault P., 2014. A simulation fidelity assessment framework. International Conference on Simulation and Modeling Methodologies, Technologies and Applications, pages 463-471, Vienne, Austria.

Roza M., 1999. Fidelity Requirements Specification: A Process Oriented View. Fall Simulation Interoperability Workshop.
Vu, L.H., Foures, D., Albert, V., 2015, ProDEVS: an eventdriven modeling and simulation tool for hybrid systems using state diagrams, Proceedings of the 8th International Conference on Simulation Tools and Techniques, 29-37, Greece.

Zeigler, B.P., Praehofer, H., Tag, G.K., 2000, Theory of modeling and simulation, San Diego, California, USA. Academic Press. 